\title{
Identification of Structural Elements that Regulate Signaling Bias in D2-like Dopamine Receptors
} David R. Sibley ${ }^{1}$, Marta Sanchez Soto ${ }^{1}$, Blair K. A. Willette ${ }^{1}$, Elizabeth C. Gonye ${ }^{1}$, Annah M. Moore ${ }^{1}$,
Amy E. Moritz ${ }^{1}$, Ravi Kumar Verma ${ }^{2}$, Hideaki Yano ${ }^{2}$, Lei Shi ${ }^{2}$, R Benjamin Free $^{1}$

${ }^{I}$ Molecular Neuropharmacology Section, National Institute of Neurological Disorders \& Stroke, National Institutes of Health, USA, ${ }^{2}$ Computational Chemistry and Molecular Biophysics Unit, National Institute on Drug Abuse, National Institutes of Health, USA

The D2 dopamine (DA) receptor (D2R) signals through multiple second messenger pathways making it difficult to discern which are linked to specific effects of D2R-targeted drugs; however, this complexity provides an opportunity to develop pathway-selective therapeutics. Structure-activity analyses and molecular modeling using the D2R G proteinbiased agonist, MLS1547, led to a structural model for biased signaling that entails a hydrophobic binding pocket formed by residues I184, F189, and V190 at the interface between the fifth transmembrane segment (TM5) and the second extracellular loop of the D2R. To investigate the role of these residues in regulating signaling by the D2-like receptors (D2R, D3R, and D4R), and the $\beta$ 2-adrenergic receptor (B2R), we constructed point mutations at I184, V190 and F189 of the D2R, and at the aligned residues for F189 (i.e., position 5.38) within the D3R, D4R, and B2R, and studied their effects on $\mathrm{G}$ protein-mediated signaling, $\beta$-arrestin recruitment, and GRK2 interactions using BRET-based biosensors and in vitro signaling assays. The D2R point mutations I184A and V190A produced a small change in the potency of DA for stimulating $\beta$-arrestin recruitment or $\mathrm{G}$ protein activation. Strikingly, the F189A mutation ablated the ability of DA and other D2R agonists to recruit $\beta$-arrestin while $\mathrm{G}$ protein-signaling efficacy was maintained. Further, the D2R F189A mutant was unable to directly interact with GRK2 and failed to internalize from the cell surface following DA stimulation. In addition, we found that mutating the residues in the D3R, D4R, and B2R at position 5.38 to Ala resulted in parallel findings (i.e., loss of agonist-stimulated $\beta$-arrestin recruitment, but minimal to no effects on $G$ protein-mediated signaling). These data demonstrate that the D2R F189A mutant, and similarly mutated D3R, D4R, and B2R, are highly biased towards $\mathrm{G}$ protein-mediated signaling and suggest that the presence of a Phe or Tyr residue at this position (5.38) is important for stabilizing an activated state for recruiting $\beta$-arrestin. Conformational changes propagated through TM5 might thus act as a molecular switch for receptor signaling via $\beta$-arrestin recruitment. These results may have implications for the design of novel signaling-biased compounds for the treatment of GPCR-related disorders. 